\title{
Current-driven ferromagnetic resonance, mechanical torques, and rotary motion in magnetic nanostructures
}

\author{
Alexey A. Kovalev, ${ }^{1,2}$ Gerrit E. W. Bauer, ${ }^{2}$ and Arne Brataas ${ }^{3,4}$ \\ ${ }^{1}$ Department of Physics, Texas A\&M University, College Station, Texas 77843-4242, USA \\ ${ }^{2}$ Kavli Institute of NanoScience, Delft University of Technology, 2628 CJ Delft, The Netherlands \\ ${ }^{3}$ Department of Physics, Norwegian University of Science and Technology, NO-7491 Trondheim, Norway \\ ${ }^{4}$ Center for Advanced Study at the Norwegian Academy of Science and Letters, Drammensveien 78, NO-0271 Oslo, Norway
}

(Received 11 September 2006; revised manuscript received 16 November 2006; published 24 January 2007)

\begin{abstract}
We study theoretically the detection and possible utilization of electric current-induced mechanical torques in ferromagnetic-normal-metal heterostructures generated by spin-flip scattering or the absorption of transverse spin currents by a ferromagnet. To this end, we analyze the dc voltage signals over a spin valve driven by an ac current. In agreement with recent studies, this "rectification," measured as a function of ac frequency and applied magnetic field, contains important information on the magnetostatics and magnetodynamics. Subsequently, we show that the vibrations excited by spin-transfer to the lattice can be detected as a splitting of the dc voltage resonance. Finally, we propose a concept for a spin-transfer-driven electric nanomotor based on integrating metallic nanowires with carbon nanotubes, in which the current-induced torques generate a rotary motion.
\end{abstract}

DOI: $10.1103 /$ PhysRevB.75.014430

PACS number(s): 76.50.+g, 72.25.Ba, 85.85. $+\mathrm{j}$

\section{INTRODUCTION}

An electromotor is an apparatus that generates rotational motion with electrical currents, as demonstrated by Faraday in 1821. Under miniaturization, the torques generated by Ørsted magnetic fields scale unfavorably compared to the increased friction, rendering the Faraday motor an unfeasible concept at the nanoscale. Alternative concepts for nanomachines are based on electrostatic forces, ${ }^{1}$ thermal fluctuations, ${ }^{2}$ torques induced by circularly polarized light, ${ }^{3}$ and angular momentum transfer by spin-polarized currents. $^{4-7}$ In this paper, we elaborate on this last idea.

A spin-polarized current carries an angular momentum current,

$$
T_{\mathrm{SP}}=P I \hbar /(2 e)=P T_{0},
$$

where $P=\left(I_{\uparrow}-I_{\downarrow}\right) / I$ is the polarization of the charge current $I=I_{\uparrow}+I_{\downarrow}, e$ is the electron charge, and $T_{0}=I \hbar /(2 e)$. If transferred completely to the lattice, mechanical torques are created of the same magnitude, which can be relatively large in nanoscale structures. Since spin currents are routinely excited in magnetoelectronic devices such as spin valves, we pose here the question of whether current-induced mechanical torques can be detected and utilized in such structures. We conclude that the resonant magnetomechanical coupling studied earlier ${ }^{8,6}$ should indeed be observable in spin-valve structures, paving the way for applications such as highfrequency actuators and transducers of mechanical motion. Furthermore, we propose a design for a spin-transfer driven electric nanomotor based on carbon nanotubes. ${ }^{3}$

The resonant rectification of a current in a spin valve as a function of an applied ac frequency has been found experimentally to form a rich source of information about the magnetization dynamics in spin-valve structures. ${ }^{9,10}$ Kupferschmidt et al. found theoretically that the spin-pumping by the magnetization dynamics ${ }^{11}$ significantly modifies these spectra. ${ }^{35}$ We suggest that the ac-dc conversion in spin valves can be used to detect vibrational modes excited by the spinpolarized currents.

In this paper, we address the theory of spin valves excited by ac currents, show how to include the effects of the magnetovibrational coupling, and predict signatures of the current-induced mechanical torques. We also share our ideas how these torques could drive a rotary (rather than vibrational) motion, i.e., an electric nanomotor. The manuscript is organized as follows. In Sec. II, we calculate (position-dependent) mechanical torques generated by the spin-flip dissipation of a spin current injected by a ferromagnet into a normal metal. In Sec. III, we study the spin-transfer mechanical torques resulting from the absorption of transverse spin currents by a ferromagnet. We suggest to employ the recently reported diode effect ${ }^{9,10}$ in $\mathrm{F}$ (erromagnet) $\mid \mathrm{N}$ (ormal) $\mid \mathrm{F}$ (erromagnet) metal spin valves to detect the vibrations created by spin-transfer mechanical torques. We calculate the nonlinear dc voltage emanating from the spin-transfer driven ferromagnetic resonance with and without the resonant magnetovibrational coupling. Spintransfer torques noncollinear with planes formed by a principal axis of anisotropies can deform the resonant line shape of dc voltage as a function of the ac current bias frequency. In Appendixes B and $\mathrm{C}$, we calculate the extra dc voltage caused by spin-pumping and prove that it can play a key role in sufficiently thin films. In Appendix B, we also conclude that the magnetovibrational coupling is observable by virtue of the spin-pumping even in asymmetric $\mathrm{N}|\mathrm{F}| \mathrm{N}$ heterostructures. In Sec. IV, we propose a spin-transfer driven nanomotor concept based on integrating metallic nanowires with carbon nanotubes.

\section{MECHANICAL TORQUES DUE TO DISSIPATION OF SPIN CURRENTS}

Consider a normal-metal diffusive wire or nanostructured pillar into which a spin accumulation has been injected via an electrically biased ferromagnetic contact (Fig. 1). Spin $\mathbf{I}_{s}$ 


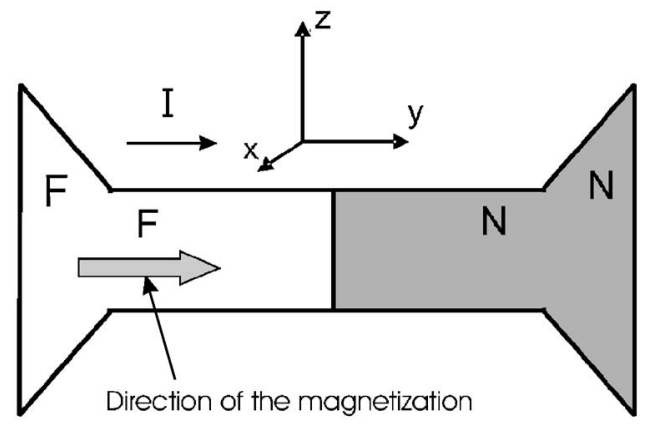

FIG. 1. Spin transfer from spin-polarized currents to the lattice in a heterostructure consisting of two (ferromagnetic and normal metal) sections connected to mechanically clamped ferromagnetic and normal metal reservoirs. Polarized currents lead to torques twisting the middle section.

and charge $I_{0}$ currents can be conveniently related via $2 \times 2$ matrices in Pauli spin space $\hat{I}=\left(\hat{1} I_{0}+\hat{\sigma} \cdot \mathbf{I}_{s}\right) / 2$, where $\hat{\sigma}$ is the vector or Pauli spin matrices and $\hat{1}$ is the $2 \times 2$ unit matrix. Spin-orbit interactions or magnetic impurities cause spin-flip scattering that can be parametrized by a spin-flip relaxation time $t_{s f}$. $\boldsymbol{\mu}_{s}$, the local (vector) spin accumulation, is related to the spin current density $\mathbf{j}_{s}=\mathbf{I}_{s} /(e S)$ ( $e$ is the electron charge and $S$ is the cross section of the wire) by the angular momentum conservation law,

$$
\frac{\partial}{\partial t} \boldsymbol{\mu}_{s}+\frac{\partial}{\partial y} \frac{\mathbf{j}_{s}}{\mathcal{N}}=-\frac{\boldsymbol{\mu}_{s}}{t_{s f}}
$$

The dissipated angular momentum per unit length, $\tau$ $=(\hbar / 2) S \mathcal{N} \boldsymbol{\mu}_{s} / t_{s f}$, where $\mathcal{N}$ is the density of states at the Fermi level, is transferred as a mechanical torque to the lattice. In the configuration sketched in Fig. 1, the injected spin accumulation $\left|\mu_{s}\right|=\mu_{s}$ and the mechanical torque are polarized in the $y$ direction. Newton's law for the mechanical motion of the substrate then reads ${ }^{12}$

$$
\rho I \frac{\partial^{2} \varphi(y, t)}{\partial t^{2}}=C \frac{\partial^{2} \varphi(y, t)}{\partial y^{2}}+\frac{\hbar}{2} S \mathcal{N} \frac{\mu_{s}(y, t)}{t_{s f}},
$$

where $\varphi(y, t)$ is the angle of torsion, $I=I_{x}+I_{z}\left(I_{z}=\int x^{2} d z d x\right.$, $\left.I_{z}=\int z^{2} d z d x\right)$ is the moment of inertia of the cross section at $y$ relative to its center of mass, $\rho$ is the mass density, and $C$ is an elastic constant defined by the shape and material of the wire $\left(C=\mu R^{4} / 2\right.$ for a circular cross section with radius $R, \mu$ is the Lamé constant). Equation (3) must be complemented by the boundary conditions $(\varphi=0)$ at the clamping points.

We now concentrate on a bimetal wire consisting of a ferromagnetic and a normal metal (Fig. 1). We assume for simplicity here that the bulk resistances of the wires are much larger than the interface resistance (Ref. 14 shows how interfaces can be taken into account) to the extent that we may disregard the latter. The magnetization and mechanical motion is much slower than the relaxation scattering, therefore we can consider only the parametrically stationary limit. The charge current density $j_{0}$ is conserved $\left(\partial_{y} j_{0}=0\right)$ and Eq. (2) reduces to

$$
-\frac{\partial}{\partial y} \mathbf{j}_{s}=\frac{\mathcal{N} \boldsymbol{\mu}_{s}}{t_{s f}}=\frac{2 \boldsymbol{\tau}}{\hbar S}
$$

In the normal metal, charge and spin currents are governed by Fick's laws $j^{N}=-\mathcal{N}^{N} D^{N} \partial_{y} \mu_{0}^{N}$ and $\mathbf{j}_{s}^{N}=-\mathcal{N}^{N} D^{N} \partial_{y} \boldsymbol{\mu}_{s}^{N}$, respectively, where $D^{N}$ is the diffusion constant and the index $N$ indicates the normal metal, leading to the diffusion equations,

$$
\frac{\partial^{2}}{\partial y^{2}} \mathcal{N}^{N} D^{N} \mu_{0}^{N}=0, \quad \frac{\partial^{2}}{\partial y^{2}} \mathcal{N}^{N} D^{N} \mu_{s}^{N}=\mathcal{N}^{N} \mu_{s}^{N} / t_{s f}^{N}=\frac{\tau_{N}}{\frac{\hbar}{2} S},
$$

where $\tau_{N}$ is the mechanical torque per unit length for the normal metal. In a ferromagnet $(F)$, the particle and spin currents are $j^{F}=-\left(\mathcal{N}_{\uparrow}^{F} D_{\uparrow}^{F} \partial_{y} \mu_{\uparrow}+\mathcal{N}_{\downarrow}^{F} D_{\downarrow}^{F} \partial_{y} \mu_{\downarrow}\right) / 2$ and $\mathbf{j}_{s}^{F}=$ $-\mathbf{m} \partial_{y}\left(\mathcal{N}_{\uparrow}^{F} D_{\uparrow}^{F} \mu_{\uparrow}-\mathcal{N}_{\downarrow}^{F} D_{\downarrow}^{F} \mu_{\downarrow}\right) / 2$, where $D_{\uparrow(\downarrow)}^{F}$ is the diffusion constants for spin-up (-down) electrons and $\mathcal{N}_{\uparrow(\downarrow)}^{F}$ is the corresponding spin-up (-down) density of states. The diffusion equation in a ferromagnet then reads

$$
\begin{aligned}
\frac{\partial^{2}}{\partial y^{2}}\left(\mathcal{N}_{\uparrow}^{F} D_{\uparrow}^{F} \mu_{\uparrow}+\mathcal{N}_{\downarrow}^{F} D_{\downarrow}^{F} \mu_{\downarrow}\right) & =0, \quad \frac{\partial^{2}}{\partial y^{2}}\left(\mathcal{N}_{\uparrow}^{F} D_{\uparrow}^{F} \mu_{\uparrow}-\mathcal{N}_{\downarrow}^{F} D_{\downarrow}^{F} \mu_{\downarrow}\right) \\
& =\mathcal{N}^{F}\left(\mu_{\uparrow}-\mu_{\downarrow}\right) / t_{s f}^{F},
\end{aligned}
$$

leading to

$$
\frac{\partial^{2}}{\partial y^{2}} D^{F}\left(\mu_{\uparrow}-\mu_{\downarrow}\right)=\left(\mu_{\uparrow}-\mu_{\downarrow}\right) / t_{s f}^{F}=\tau_{F} \prime\left[\frac{\hbar}{2} S \mathcal{N}^{F}\right],
$$

where $\tau_{F}$ is the mechanical torques per unit length for the ferromagnetic metal, $D^{F}=2 D_{\uparrow}^{F} D_{\downarrow}^{F} \mathcal{N}_{F} /\left(\mathcal{N}_{\uparrow}^{F} D_{\uparrow}^{F}+\mathcal{N}_{\downarrow}^{F} D_{\downarrow}^{F}\right)$, and $\mathcal{N}^{F}=\left(\mathcal{N}_{\uparrow}^{F}+\mathcal{N}_{\downarrow}^{F}\right) / 2$.

We can now calculate the mechanical torques by solving the diffusion equations, requiring the continuity of distribution functions and conservation of the spin and charge currents at the interface. ${ }^{13,14} \mathrm{We}$ also require no spin accumulation at the connection with reservoirs. For the ferromagnetic $(y<0)$ and normal $(y>0)$ metals we find, respectively,

$$
\begin{aligned}
& \tau_{F}(y)=T_{0} \frac{P \sinh \left[\left(L_{F}+y\right) / l_{s d}^{F}\right] / \sinh \left(L_{F} / l_{s d}^{F}\right)}{l_{s d}^{F} \operatorname{coth}\left(L_{F} / l_{s d}^{F}\right)+v l_{s d}^{N} \operatorname{coth}\left(L_{N} / l_{s d}^{N}\right)}, \\
& \tau_{N}(y)=T_{0} \frac{v P \sinh \left[\left(L_{N}-y\right) / l_{s d}^{N}\right] / \sinh \left(L_{N} / l_{s d}^{N}\right)}{l_{s d}^{F} \operatorname{coth}\left(L_{F} / l_{s d}^{F}\right)+v l_{s d}^{N} \operatorname{coth}\left(L_{N} / l_{s d}^{N}\right)},
\end{aligned}
$$

where $P=\left(G_{\uparrow}-G_{\downarrow}\right) /\left(G_{\uparrow}+G_{\downarrow}\right)$ is the current polarization of the ferromagnet defined here in terms of the spin-up and spin-down conductances $G_{\uparrow}$ and $G_{\downarrow}, l_{s d}^{F}=\sqrt{D^{F} t_{s f}^{F}}$ and $l_{s d}^{N}$ $=\sqrt{D^{N} t_{s f}^{N}}$ are the spin-diffusion lengths in the ferromagnet and normal metals, respectively, $T_{0}$ has been introduced in Eq. (1), $L_{F}$ and $L_{N}$ are the lengths of the ferromagnet and normal metals, respectively, and $v=\left[\mathcal{N}_{N} t_{s f}^{F}\right] /\left[\mathcal{N}_{F} t_{s f}^{N}\right]$. For the configuration sketched in Fig. 1, the mechanical torque is directed along the $y$ axis. The torque density is discontinuous when $v \neq 1$. As shown in Fig. 2, the mechanical torques are enhanced close to the $\mathrm{F} \mid \mathrm{N}$ interface on the scale defined by the spin-diffusion length in both ferromagnet and normal 


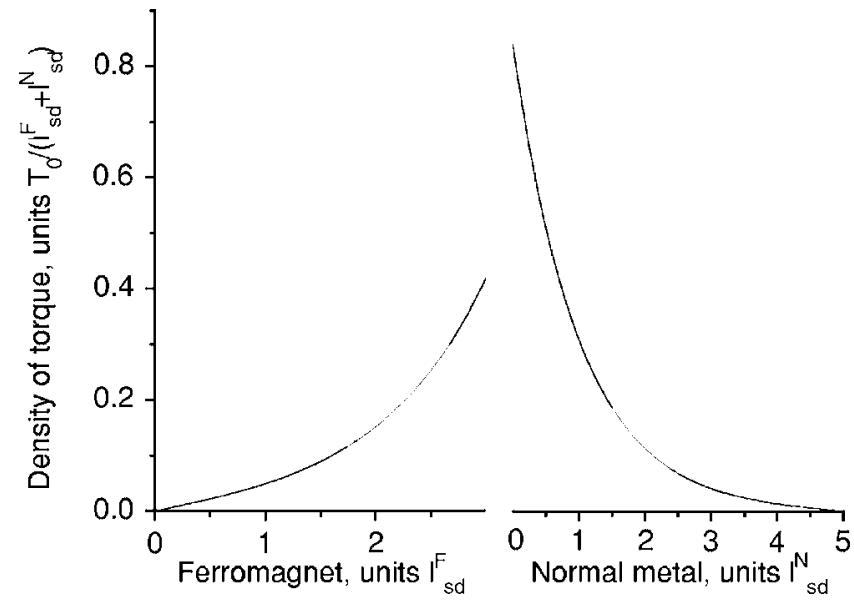

FIG. 2. The mechanical torque per unit length along the $\mathrm{F} \mid \mathrm{N}$ wire; $P=0.7, l_{s d}^{F}=10 \mathrm{~nm}, l_{s d}^{N}=20 \mathrm{~nm}, L_{F} / l_{s d}^{F}=3, L_{N} / l_{s d}^{N}=5$, and $v$ $=2$.

metals. The mechanical torques change sign with the electric currents direction.

Integrating Eqs. (5) and (6) leads to the total torque acting on the wire,

$$
\begin{aligned}
& T_{F}=T_{0} \frac{P l_{s d}^{F} \tanh \left(L_{F} / 2 l_{s d}^{F}\right)}{l_{s d}^{F} \operatorname{coth}\left(L_{F} / l_{s d}^{F}\right)+v l_{s d}^{N} \operatorname{coth}\left(L_{N} / l_{s d}^{N}\right)}, \\
& T_{N}=T_{0} \frac{v P l_{s d}^{N} \tanh \left(L_{N} / 2 l_{s d}^{N}\right)}{l_{s d}^{F} \operatorname{coth}\left(L_{F} / l_{s d}^{F}\right)+n l_{s d}^{N} \operatorname{coth}\left(L_{N} / l_{s d}^{N}\right)} .
\end{aligned}
$$

When $L_{F} \gg l_{s d}^{F}$ and $L_{N} \gg l_{s d}^{N}$, we obtain $T_{\mathrm{SP}}=T_{F}+T_{N}=P \tau_{0}$ as expected by the complete dissipation of the spin current in this limit [Eq. (1)]. By ultrasensitive displacement detection, it should be possible to observe the mechanical strain caused by the spin-flip torques ${ }^{4}$ in the setup of Fig. 1.

\section{GENERATION AND DETECTION OF THE MECHANICAL TORQUES DUE TO SPIN TRANSFER}

In the previous section, we studied mechanical torques arising from spin-flip relaxation processes within the bulk of the metals. In contrast, in this section, we will consider structures (see Fig. 3) in which the spin transfer is dominated by dephasing processes (leading to absorption of transversely polarized currents) ${ }^{13}$ at the normal metal|ferromagnetic interfaces, whereas the spin-flip processes in the bulk materials are disregarded (which can be repaired easily if necessary). Such a device is superior to the wires of the previous section in generating mechanical torques when the structures are smaller than the spin-diffusion length. We propose here to induce and detect the magnetovibrational modes ${ }^{8}$ driven by spin-transfer torques in devices such as that shown in Fig. 3, i.e., a doubly clamped heterostructure in the shape of a bar with a ferromagnetic load in the center. In our setup, the mechanical torque is generated in the ferromagnet $\mathrm{F} 2$ by the transversely polarized spin current (ferromagnet F1 is supposed to be clamped by the substrate). The absorbed spinangular momentum is transferred to the lattice by the mag-

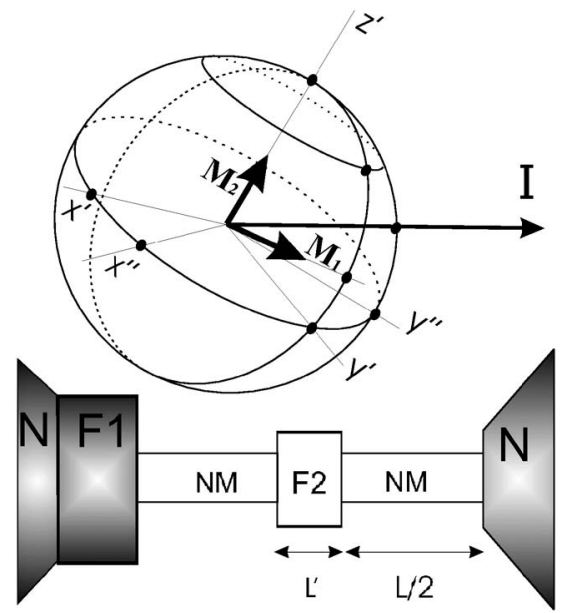

FIG. 3. Model sample to study spin transfer to the lattice consisting of ferromagnets F1 and F2 (length $L^{\prime}$ ) and NM (length $L / 2$, i.e., tunnel junctions or normal metal). $\mathrm{F} 1$ and the normal metal $\mathrm{N}$ are electron reservoirs that are mechanically clamped. The vector diagram shows the reference frames used in calculations with respect to the magnetizations. The magnetization of F1 is fixed, $\mathbf{M}_{1}$ $\equiv \mathbf{M}_{\text {fixed }}$, and that of $\mathrm{F} 2$ is variable, $\mathbf{M}_{2} \equiv \mathbf{M}$.

netic shape and crystal anisotropies. In the regime of the resonant magnetovibrational coupling, the mechanical motion is strongly affected by the magnetization dynamics created by spin-transfer torques, ${ }^{8}$ which, in turn, affect the transport properties. We predict that such vibrations should be detectable by current-driven ferromagnetic resonance experiments reported in Refs. 9 and 10. We start by analyzing the dc voltage signals over a spin valve resulting from the "rectification" of an ac current by the precessing magnetization (without magnetovibrational coupling, e.g., setups from Refs. 9 and 10) as an intermediate step. A general analysis of the dc signals in the regime of the resonant magnetovibrational coupling is subsequently given. We also estimate the maximum torques that can be created in these structures.

The derivation below is carried out for a system in which the ferromagnet F2 is inserted into the normal metal island. However, the rectification of the ac current and the excitation of magnetovibrational modes also occur (and can be treated by our methods) in a system in which the ferromagnet is attached to the side or on top of the normal metal wire ("spin-flip transistor"). ${ }^{15,16,36}$

\section{A. Ferromagnetic resonance driven by spin-transfer torques}

Initially, we assume that the mechanical modes are not excited in the device depicted in Fig. 3. One (the "hard") layer magnetization of F1 is assumed completely fixed by shape or crystal anisotropies to the direction $\mathbf{M}_{1}=\mathbf{M}_{\text {fixed }}$ with unit vector $\mathbf{m}_{\text {fixed. }}$. We are interested in the dynamics of the magnetization direction $\mathbf{m}=\mathbf{M}_{2} / M_{s}$ of the middle ("soft") layer F2, where $\left|\mathbf{M}_{2}\right|=M_{s}$ is the constant saturation magnetization, in the presence of an ac electric current bias. We consider the soft layer to be at resonance, ${ }^{10}$ but note that the soft and hard layers can exchange their roles as a function of frequency (e.g., in Fig. 5). We model the magnetization dy- 
namics by the generalized Landau-Lifshitz-Gilbert (LLG) equation in the macrospin approximation that is augmented by the current-dependent spin-transfer torque, ${ }^{17}$

$$
\begin{aligned}
\frac{d \mathbf{M}}{d t}= & -\gamma \mathbf{M} \times \mathbf{H}_{\text {eff }}+\frac{\alpha}{M_{s}} \mathbf{M} \times\left(\frac{d \mathbf{M}}{d t}\right)+\gamma \frac{\hbar}{2 e} \frac{I(t)}{V_{m}}\left[\eta_{1} \mathbf{m}\right. \\
& \left.\times\left(\mathbf{m}_{\text {fixed }} \times \mathbf{m}\right)+\eta_{2}\left(\mathbf{m}_{\text {fixed }} \times \mathbf{m}\right)\right],
\end{aligned}
$$

where $I(t)$ is the time-dependent current through the system, $\eta_{1}$ describes the efficiency of conventional spin transfer, and $\eta_{2}$ parametrizes an "effective spin-transfer exchange field." When the spacer is an insulator, as in the experiment of the Tsukuba group, ${ }^{9}$ the parameter $\eta_{1}$ is a constant governed by the expressions such as derived by Slonczewski. ${ }^{18}$ The "effective field" $\eta_{2}$ has been less well investigated in magnetic tunnel junctions and is treated here as an adjustable parameter. When the spacer is a normal metal in a configuration sketched in Fig. 3, the LLG equation for the soft layer reads

$$
\begin{aligned}
\frac{d \mathbf{M}}{d t}= & -\gamma \mathbf{M} \times \mathbf{H}_{\mathrm{eff}}+\frac{\alpha}{M_{s}} \mathbf{M} \times\left(\frac{d \mathbf{M}}{d t}\right) \\
& -\frac{\gamma \hbar}{2 e V_{m}} \mathbf{m} \times\left[\left(\mathbf{I}_{s 1}+\mathbf{I}_{s 2}\right) \times \mathbf{m}\right],
\end{aligned}
$$

where the spin currents leaving the soft ferromagnet can be computed by magnetoelectronic circuit theory ${ }^{13}$ as

$$
\begin{aligned}
I_{1(2)}=\left(G_{\uparrow}\right. & \left.G_{\downarrow}\right)\left(\mu_{0}^{2(1)}-\mu_{0}^{1(2)}\right)+\left(G_{\uparrow}-G_{\downarrow}\right)\left(\boldsymbol{\mu}_{s}^{2(1)}-\boldsymbol{\mu}_{s}^{1(2)}\right) \cdot \mathbf{m}, \\
\mathbf{I}_{s 1(2)}= & \mathbf{m}\left[\left(G_{\uparrow}-G_{\downarrow}\right)\left(\mu_{0}^{2(1)}-\mu_{0}^{1(2)}\right)+\left(G_{\uparrow}+G_{\downarrow}\right)\left(\boldsymbol{\mu}_{s}^{2(1)}\right.\right. \\
& \left.\left.-\boldsymbol{\mu}_{s}^{1(2)}\right) \cdot \mathbf{m}\right]-\left(2 \mathbf{m} \times \boldsymbol{\mu}_{s}^{1(2)}\right) \times \mathbf{m} G_{r}-(2 \mathbf{m} \\
& \left.\times \boldsymbol{\mu}_{s}^{1(2)}\right) G_{i},
\end{aligned}
$$

where $\alpha$ is the Gilbert damping constant, $\gamma$ is the gyromagnetic ratio, $G_{\uparrow}$ and $G_{\downarrow}$ describe the conventional spindependent conductances limited by bulk and interface scattering and $G_{\uparrow \downarrow}=G_{r}+i G_{i}$ is the interface mixing conductance of the ferromagnet, $\mu_{0}$ and $\boldsymbol{\mu}_{s}$ are the chemical potential and spin-accumulation in the normal metals, respectively, and the spin $\mathbf{I}_{s 1(2)}$ and charge $I_{1(2)}$ currents correspond to the current (spin) flow into the normal metal node 1 (2). It is shown in Appendix A that Eqs. (9) and (10) are equivalent when we allow for an angle dependence of the parameters $\eta_{1(2)}$. The efficiencies of the spin-transfer torque $\eta_{1}(\theta)$ (Ref. 14) and the "effective spin-transfer field" $\eta_{2}(\theta)$ mainly depend on the real and imaginary part of the mixing conductance, respectively.

Choosing the $z^{\prime}$ axis along the equilibrium direction $\left(\mathbf{M}_{0}\right)$ of the soft layer and the $x^{\prime}$ axis perpendicular to both magnetizations (see Fig. 3), we expand the free energy close to the equilibrium direction of the magnetization as

$$
F(\mathbf{M})=F\left(\mathbf{M}_{0}\right)+N_{x^{\prime}} M_{x^{\prime}}^{2} / 2+N_{y^{\prime}} M_{y^{\prime}}^{2} / 2+N_{x^{\prime} y^{\prime}} M_{x^{\prime}} M_{y^{\prime}}
$$

such that the effective magnetic field

$$
\begin{aligned}
\mathbf{H}_{\mathrm{eff}}=-\partial F / \partial \mathbf{M}= & -\left(N_{x^{\prime}} M_{x^{\prime}}+N_{x^{\prime} y^{\prime}} M_{y^{\prime}}\right) \mathbf{x}^{\prime} \\
& -\left(N_{y^{\prime}} M_{y^{\prime}}+N_{x^{\prime} y^{\prime}} M_{x^{\prime}}\right) \mathbf{y}^{\prime},
\end{aligned}
$$

where $N_{x^{\prime}}, N_{y^{\prime}}$, and $N_{x^{\prime} y^{\prime}}$ are parameters characterizing the energy of the macrospin excitations. The free energy Eq. (13) is not diagonal on the basis of the magnetizations along the $x^{\prime}$ and $y^{\prime}$ axis, but can be diagonalized in another basis rotated by the angle $\phi$ around the $z^{\prime}$ axis, so that $N_{x^{\prime}}$ $=N_{x}^{d} \cos ^{2} \phi+N_{y}^{d} \sin ^{2} \phi, N_{y^{\prime}}=N_{y}^{d} \cos ^{2} \phi+N_{x}^{d} \sin ^{2} \phi$, and $N_{x^{\prime} y^{\prime}}$ $=\left(N_{x}^{d}-N_{y}^{d}\right) \cos \phi \sin \phi$. Here, $N_{x}^{d}$ and $N_{y}^{d}$ are the eigenvalues of the expansion tensor in Eq. (13). We consider here an arbitrary direction of an external magnetic field $\mathbf{H}_{0}$. In general, the components of $\mathbf{H}_{0}$ contribute to Eq. (13) in a nontrivial way. As an illustration, let us consider an external field along the $z$ axis, which can be included as follows: $N_{x^{\prime}}$ $=N_{x^{\prime}}^{0}+H_{0} / M_{s}$ and $N_{y^{\prime}}=N_{y^{\prime}}^{0}+H_{0} / M_{s}$, where $N_{x(y)}^{0}$ are elements of the magnetic anisotropy tensor.

The linear response of the magnetization to an ac current perturbation is given by the response functions $\chi_{x^{\prime} I}$ $=\left(M_{x^{\prime}} / I\right)_{\omega}$ and $\chi_{y^{\prime} I}=\left(M_{y^{\prime}} / I\right)_{\omega}$. From Eqs. (9) and (14),

$$
\chi_{x^{\prime} I}(\omega)=\frac{\hbar \gamma \sin \theta}{2 e V_{m}} \frac{i \eta_{2} \omega+\gamma M_{s} \Gamma_{x^{\prime}}}{\omega^{2}\left(1+\alpha^{2}\right)-\omega_{m}^{2}+2 i \alpha^{\prime} \omega \omega_{m}},
$$

$$
\chi_{y^{\prime} I}(\omega)=\frac{\hbar \gamma \sin \theta}{2 e V_{m}} \frac{-i \eta_{1} \omega+\gamma M_{s} \Gamma_{y^{\prime}}}{\omega^{2}\left(1+\alpha^{2}\right)-\omega_{m}^{2}+2 i \alpha^{\prime} \omega \omega_{m}},
$$

where $V_{m}$ is the volume of the magnet, $\omega_{m}^{2}=\gamma^{2} N_{x}^{d} N_{y}^{d} M_{s}^{2}$ $=\gamma^{2} M_{s}^{2}\left(N_{x^{\prime}} N_{y^{\prime}}-N_{x^{\prime} y^{\prime}}^{2}\right), \quad \Gamma_{x^{\prime}}=\eta_{1}\left(N_{y^{\prime}}+N_{x^{\prime} y^{\prime}} \alpha\right)+\eta_{2}\left(N_{x^{\prime} y^{\prime}}\right.$ $\left.-N_{y^{\prime}} \alpha\right), \quad \Gamma_{y^{\prime}}=\eta_{1}\left(N_{x^{\prime} y^{\prime}}+N_{x^{\prime}} \alpha\right)+a_{2}\left(N_{x^{\prime}}-N_{x^{\prime} y^{\prime}} \alpha\right), \quad$ and the damping parameter is modified by the anisotropies as

$$
\alpha^{\prime}=\alpha \frac{\left(N_{x}^{d}+N_{y}^{d}\right) / 2}{\sqrt{N_{x}^{d} N_{y}^{d}}}=\alpha \frac{\left(N_{x^{\prime}}+N_{y^{\prime}}\right) / 2}{\sqrt{N_{x^{\prime}} N_{y^{\prime}}-N_{x^{\prime} y^{\prime}}^{2}}} .
$$

From Eqs. (15) and (16), we conclude that both the field effect $\eta_{2}$, characteristic for tunnel junctions, ${ }^{9}$ and noncollinear anisotropies $N_{x^{\prime} y^{\prime}}$ cause a phase shift in the magnetization response $\chi_{y^{\prime} I}(\omega)$ that is relevant to the rectification effect, as demonstrated below. At resonance, the parameters $\Gamma_{x^{\prime}}$ and $\Gamma_{y^{\prime}}$ are the components of the out-of-phase magnetization response.

The resulting magnetization dynamics causes oscillations of the magnetoresistance $R\left(\mathbf{m}_{1}(t), \mathbf{m}_{2}(t)\right)=R[\cos \theta(t)]$ of the multilayer structure (details of the calculations are given in Appendix A). In the presence of an ac current bias $I(t)$ $=I_{0} \operatorname{Re} e^{i \omega t}$, the resistance has an oscillating component that in the linear approximation reads

$$
R[\cos \theta(t)] \approx R(\nu)-\sin \theta \frac{\partial R(\nu)}{\partial \nu} \Delta \theta(t)
$$




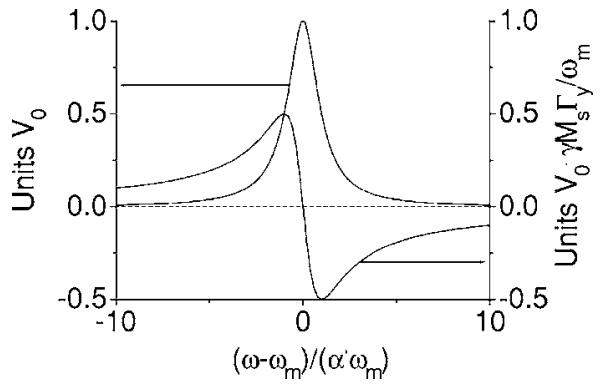

FIG. 4. The dimensionless dc voltage over the sample biased by an ac current has two components that are plotted as a function of ac current frequency; $V_{0}=I_{0}^{2} \sin ^{2} \theta \frac{\partial R(\nu)}{\partial \nu} \frac{\hbar}{2 e} \frac{\gamma}{2 M_{s} V_{m}} \frac{1}{\alpha^{\prime} \omega_{m}}$.

$$
\begin{gathered}
=R(\nu)-\sin \theta \frac{\partial R(\nu)}{\partial \nu} m_{y^{\prime}}(t)=R(\nu) \\
-\frac{I_{0}}{M_{s}} \sin \theta \frac{\partial R(\nu)}{\partial \nu} \operatorname{Re}\left(e^{i \omega t} \chi_{y^{\prime} I}\right),
\end{gathered}
$$

leading to a nonlinear phase-sensitive effect in the voltage across the sample,

$$
\begin{aligned}
U=R(t) I(t)= & R(\nu) I(t)-\frac{\sin \theta}{4 M_{s}} \frac{\partial R(\nu)}{\partial \nu}\left(I_{0} e^{i \omega t} \chi_{y^{\prime} I}+I_{0} e^{-i \omega t} \chi_{y^{\prime} I}^{*}\right) \\
& \times\left(I_{0} e^{i \omega t}+I_{0} e^{-i \omega t}\right) \\
& \sim I_{0}^{2} \operatorname{Re}\left[\left(1+e^{i 2 \omega t}\right) \chi_{y^{\prime} I}\right]
\end{aligned}
$$

where the parameter $\nu=\cos \theta$ describes the equilibrium configuration of the magnetizations and $\theta(t)=\theta+\Delta \theta(t)$ (to lowest order $\left.\Delta \theta \approx m_{y^{\prime}}\right)$. The magnetization dynamics is thus manifest in the nonlinear response, i.e., the zero and secondharmonic components of the voltage across the sample,

$$
\begin{aligned}
U_{0}= & \frac{I_{0}^{2} \sin \theta}{2 M_{s}} \frac{\partial R(\nu)}{\partial \nu} \operatorname{Re} \chi_{y^{\prime} I}(\omega) \\
\begin{array}{c}
\omega \rightarrow \omega_{m} \\
=
\end{array} & -I_{0}^{2} \sin ^{2} \theta \frac{\partial R(\nu)}{\partial \nu} \frac{\hbar}{2 e} \frac{\gamma}{2 M_{s} V_{m}} \frac{1}{\omega_{m}} \\
& \times\left(\frac{\alpha^{\prime} \omega_{m}^{2}}{\left(\omega-\omega_{m}\right)^{2}+\alpha^{\prime 2} \omega_{m}^{2}}-\frac{\left(\omega-\omega_{m}\right) \gamma M_{s} \Gamma_{y^{\prime}}}{\left(\omega-\omega_{m}\right)^{2}+\alpha^{\prime 2} \omega_{m}^{2}}\right), \\
U_{2 \omega}= & \frac{I_{0}^{2} \sin \theta}{2 M_{s}} \frac{\partial R(\nu)}{\partial \nu}\left|\chi_{y^{\prime} I}(\omega)\right| \\
\omega \rightarrow \omega_{m} & =I_{0}^{2} \sin ^{2} \theta \frac{\partial R(\nu)}{\partial \nu} \frac{\hbar}{2 e} \frac{\gamma}{2 V_{m}} \times \sqrt{\frac{1+\gamma^{2} M_{s}^{2} \Gamma_{y^{\prime}} / \omega^{2}}{\left(\omega-\omega_{m}\right)^{2}+\alpha^{\prime 2}}} .
\end{aligned}
$$

As pointed out in Ref. 9, the dc voltage $U_{0}$ can be interpreted as a diodelike rectification. The amplitudes $U_{0}$ and $U_{2 \omega}$ show a resonant enhancement close to $\omega_{m}$ (note that $U_{0}$ corresponds to $V_{\text {mix }}$ in Ref. 10 and its sign corresponds to a current flow from soft to hard ferromagnetic layer). Equation (22) is a linear combination of the symmetric and antisym-

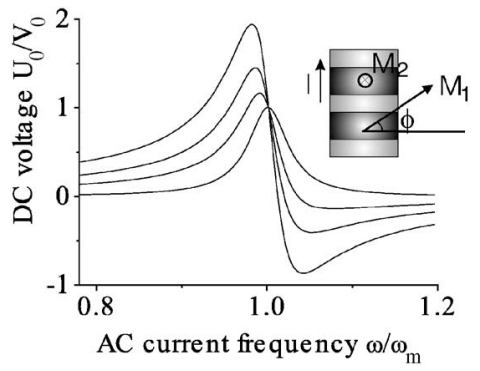

FIG. 5. The resonance peaks are skewed when the spin-transfer torque does not lie in a plane formed by principal axes of the shape anisotropy. We illustrate this by exciting the magnetization $\mathbf{M}_{2}$ of the harder (resonant) layer with an easy plane anisotropy by ac currents, while the soft layer magnetization is forced into the directions $\phi=0: \pi / 20: \pi / 10: \pi / 4$ by an external magnetic field. Here $\eta_{2}=0$.

metric Lorentzians (see Fig. 4). By fitting the dc voltage $U_{0}(\omega)$ to a linear combination of the two curves in Fig. 4, we can determine the parameter $\Gamma_{y^{\prime}} \approx \eta_{1} N_{x^{\prime} y^{\prime}}+\eta_{2} N_{x^{\prime}}$ that is affected by the effective field $\eta_{2}$ and the noncollinear anisotropy $N_{x^{\prime} y^{\prime}}$. In Fig. 5, we demonstrate how the resonance becomes skewed merely by the noncollinear shape anisotropy (the spin-transfer torque does not lie in a plane formed by principal axes of the shape anisotropy). The resonant layer with the magnetization $\mathbf{M}_{2}$ corresponds to the harder layer in this plot (as was discussed in Sec. III A, the harder and softer layers can switch their roles with a proper choice of the ac current frequency). In the opposite scenario, ${ }^{9}$ without anisotropies, the effective field $\eta_{2}$ can still cause an antisymmetric Lorentzian signal since $\Gamma_{y^{\prime}} \approx \eta_{2} N_{x^{\prime}}=\eta_{2} H_{0} / M_{s}$, as reported by Ref. 35 .

\section{B. Magnetovibrational resonance driven and detected by spin-transfer torques}

In the following, we repeat the derivation of the previous section but allow for a torsional degree of freedom along the current flow. This requires a free-standing conducting structure, such as a doubly clamped cantilever. We already discussed the coupled dynamics of the magnetization and the lattice for a small magnet at the free tip of a cantilever that is clamped on the other side. ${ }^{8}$ However, here we consider the limit in which the magnet is heavy compared to the cantilever. This reduces the mechanical resonance frequencies, but increases the magnetomechanical coupling strength. The nor-

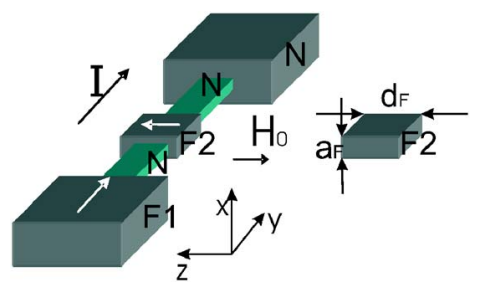

FIG. 6. (Color online) A device to detect magnetovibrational coupling caused by magnetic form and crystal anisotropies. An external magnetic field $\mathbf{H}_{0}$ is used to tune the FMR frequency. 
mal metal lattice serves as a spring and the ferromagnet is the load. We first consider a well-aligned structure for which the primed and unprimed coordinate systems in Fig. 6 coincide. A mechanical torsion profile described by the angle $\varphi(y)$ increases the free energy as

$$
\begin{aligned}
F(\mathbf{M})= & F\left(\mathbf{M}_{0}\right)+V_{m}\left\{N_{x}\left[M_{x}+M_{z} \varphi(0)\right]^{2} / 2+N_{y} M_{y}^{2} / 2\right. \\
& \left.+N_{x y}\left[M_{x}+M_{z} \varphi(0)\right] M_{y}\right\}+\frac{C}{2} \int_{-L / 2}^{L / 2}\left(\frac{\partial \varphi}{\partial y}\right)^{2} d y,
\end{aligned}
$$

where $C$ is an elastic constant defined by the shape of the cross section and the material of the normal metal link $(C$ $=\mu d a^{3} / 3$ for a long plate with thickness $a$ much smaller than width $d, a \ll d, \mu$ is the Lamé constant for the normal metal), and $\varphi(0)$ is the torsion angle at the middle magnetic section. The coefficients $N_{x}, N_{y}$, and $N_{x y}$ have the same meaning as in the previous section (one expects $N_{x y}=0$ in the setup of Fig. 6; however, we keep $N_{x y}$ in order to use our results for more general configurations). The width $L^{\prime}$ of the magnetic layer along the axis $y$ is supposed to be small compared to the length of the normal metal links $L\left(\gg L^{\prime}\right.$ ), so internal strains and deformations in the magnetic section are disregarded. The integration is therefore carried out from one clamping point $y=-L / 2$ to the other at $y=L / 2$, excluding the ferromagnetic layer. With Eq. (24), the Landau-Lifshitz-Gilbert equation has to be modified as

$$
\begin{aligned}
& \frac{d \mathbf{M}}{d t}=- \gamma \mathbf{M} \times \mathbf{H}_{\mathrm{eff}}+\frac{\alpha}{M_{s}} \mathbf{M} \times\left(\frac{d \mathbf{M}}{d t}\right)_{m}+\gamma \frac{\hbar}{2 e} \frac{I(t)}{V_{m}}\left[\eta_{1} \mathbf{m}\right. \\
& \times\left(\mathbf{m}_{\mathrm{fixed}}\right.\left.\times \mathbf{m})+\eta_{2}\left(\mathbf{m}_{\mathrm{fixed}} \times \mathbf{m}\right)\right], \\
& \mathbf{H}_{\mathrm{eff}}=-\left[N_{x} M_{x}+N_{x y} M_{y}+N_{x} M_{z} \varphi(0)\right] \mathbf{x} \\
&-\left[N_{y} M_{y}+N_{x y} M_{x}+N_{x y} M_{z} \varphi(0)\right] \mathbf{y},
\end{aligned}
$$

where the derivative $(d \mathbf{M} / d t)_{m}$ is defined in the reference frame of the magnet, since most proposed mechanisms for Gilbert damping act on the magnetization motion relative to the underlying lattice only. This can be taken into account by

$$
\left(\frac{d \mathbf{M}}{d t}\right)_{m}=\frac{d \mathbf{M}}{d t}-\frac{d \varphi(0)}{d t} M_{z} \mathbf{x} .
$$

The equation of mechanical torsional motion of the normal metal strip is ${ }^{12}$

$$
C \frac{\partial^{2} \varphi}{\partial y^{2}}=\rho I \frac{\partial^{2} \varphi}{\partial t^{2}},
$$

where $I=\int\left(z^{2}+x^{2}\right) d z d x \simeq a d^{3} / 12$ is again the moment of inertia of the cross section about its center of mass and $\rho$ is the mass density. The oscillating solution has the form $\varphi$ $=\left[A_{1} \sin (k y)+A_{2} \cos (k y)\right] e^{i \omega t}$, where $k=\omega / c$ is the wave number, $c=2 c_{t} a / d=\sqrt{C /(\rho I)}$, and $c_{t}=\sqrt{\mu / \rho}$ is the sound velocity of the transverse mode. The free constants $A_{1}$ and $A_{2}$ are determined by the boundary conditions.

The first condition $\left.\varphi\right|_{y=L / 2(-L / 2)}=0$ corresponds to perfect clamping at the boundaries. The second one, at the connection with the ferromagnetic load, can be obtained from the variational principle applied to Eq. (24), and corresponds to the torque $C \partial \varphi /\left.\partial y\right|_{y= \pm L^{\prime} / 2}$ exerted by the magnetization at the interface to the normal metal link,

$$
\left.C \frac{\partial \varphi}{\partial y}\right|_{y= \pm L^{\prime} / 2}=\left.\frac{1}{\gamma}\left(\frac{d \mathbf{M}}{d t}+\gamma \mathbf{M} \cdot \mathbf{H}_{0}\right)\right|_{y}+L^{\prime} I_{F} \rho_{F} \frac{d^{2} \varphi(0)}{d t^{2}},
$$

where $I_{F}$ is the moment of inertia of the magnetic load (for a thin plate of mass $M$, we can approximate it as $L^{\prime} I_{F} \rho_{F}$ $=M d_{F}^{2} / 12$, where $d_{F}$ is its width, see Fig. 6), and $\partial \varphi /\left.\partial y\right|_{y= \pm L^{\prime} / 2}$ is the derivative of the torsion angle of the normal metal link at the connection with the ferromagnet. This boundary condition is equivalent to the conservation of the mechanical angular momentum written for the magnetic load. With $C \partial \varphi /\left.\partial y\right|_{y= \pm L^{\prime} / 2}=C k \varphi \cot k L$, we obtain

$$
C k \varphi(0) \cot (k L)-L^{\prime} I_{F} \boldsymbol{\rho}_{F} \frac{d^{2} \varphi(0)}{d t^{2}}=\left.\frac{1}{\gamma}\left(\frac{d \mathbf{M}}{d t}+\gamma \mathbf{M} \cdot \mathbf{H}_{0}\right)\right|_{y},
$$

where the right-hand side describes the magnetically induced torques that to leading order equal the Gilbert and anisotropy torques. In the absence of the magnetic anisotropies and Gilbert damping, the two terms $d \mathbf{M} / d t$ and $\gamma \mathbf{M} \times \mathbf{H}_{0}$ cancel each other.

By only considering the left-hand side of Eq. (31), we can find the mechanical resonance frequency as $\omega_{e}$ $=\sqrt{C k \cot (k L) /\left(L^{\prime} I_{F} \rho_{F}\right)} \approx \sqrt{C /\left(L L^{\prime} I_{F} \rho_{F}\right)}$, where the approximation holds when $\omega_{e}$ is smaller than the resonant frequency of the normal metal link with a free end (which corresponds to the heavy-load limit). In frequency space, the left-hand side of Eq. (31) can be expressed in terms of the dimensionless response function $F(\omega)$ of the mechanical subsystem to an oscillating torque of frequency $\omega$ applied to the load,

$$
F(\omega)=\omega_{e}^{2} /\left[\left(\omega^{2}-\omega_{e}^{2}+2 i \beta \omega\right)\right],
$$

where $\beta$ is a phenomenological damping constant describing dissipation in Eq. (29) and it is related to the quality factor $Q$ of oscillator at the resonance frequency $\omega_{e}$ as $Q=\omega_{e} /(2 \beta)$ (at $1 \mathrm{GHz} Q \sim 500){ }^{19}$ In terms of $F(\omega)$, the mechanical response function reads

$$
(\varphi / T)_{\omega}=[1 /(C k \cot (k L))] F(\omega) \approx(L / C) F(\omega),
$$

where $T$ is the torque externally exerted on the load.

To first order in the mechanical and magnetization oscillations, Eqs. (25) and (31) lead us to the following response function:

$$
\begin{aligned}
& \chi_{y I}(\omega)=\left(M_{y} / I\right)_{\omega}=\frac{\hbar \gamma}{2 e V_{m}} \\
& \times \frac{-i \eta_{1} \omega+\Gamma_{y} \gamma\left[M_{s}+g\left(H_{0} / N_{x}\right) F(\omega)\right]}{\omega^{2}-\omega_{m}^{2}+2 i \alpha^{\prime} \omega \omega_{m}+\Lambda F(\omega)} \\
& \stackrel{g \rightarrow 0}{\approx} \frac{\hbar \sin \theta \gamma}{2 e V_{m}} \frac{-i \eta_{1} \omega+\gamma M_{s} \Gamma_{y}}{\omega^{2}-\omega_{m}+2 i \alpha^{\prime} \omega \omega_{m}+\Lambda F(\omega)},
\end{aligned}
$$

where 


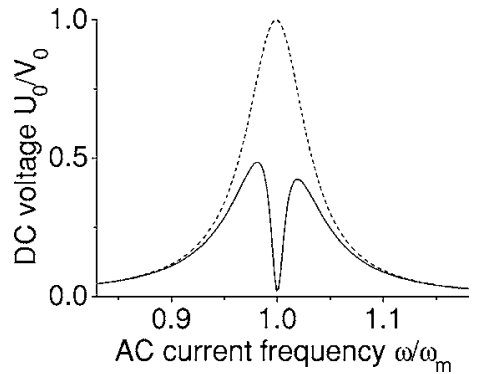

FIG. 7. Dependence of the dc component of the voltage on the frequency or the ac current bias for purely FMR (dashed line) and MVR (solid line) $\left(\omega_{m}=\omega_{e}, \alpha^{\prime}=0.02, \beta / \omega=0.002, g=0.001\right)$.

$$
\Lambda=g\left[\omega^{2}-H_{0} \omega_{m} /\left(N_{x} M_{s}\right)\right]
$$

and

$$
\begin{aligned}
g & =M_{s}^{2} V_{m} N_{x}[1 /(C k \cot (k L))] \\
& \approx M_{s}^{2} V_{m} N_{x} L / C=N_{x}(L / a)^{2}\left(V_{m} / V_{l}\right)\left(M_{s}^{2} / \mu\right) .
\end{aligned}
$$

$V_{m}$ and $V_{l}$ are the volumes of the magnetic load and the normal metal spring, respectively. $L$ and $a$ are the largest and the smallest dimensions of the normal metal links, $k=\omega_{e} / c$. We recover here the results from Ref. 6, implying that the spin-polarized current is equivalent to an external rf field along the $x$ axis applied to a magnetic cantilever. Since the ratio $V_{m} / V_{l}$ can be made much larger compared to the limit of light load considered in Ref. 6, we conclude that by making the normal metal links thinner (in the setup of Ref. 6, we have to make the cantilever thinner) we reduce the stiffness of the device, which results in a better sensitivity and stronger coupling. The reduced stiffness leads to a drop in the resonance frequency that in turn can be compensated by making the structures smaller.

The nonlinear response to an ac current can now be found by substituting Eq. (34) into the first parts of Eqs. (22) and (23). We conclude that the most conspicuous feature of the magnetovibrational coupling is the formation of a magnetopolariton and the splitting of the ferromagnetic resonance close to $\omega=\omega_{m}$, which is governed by $\sqrt{\Lambda}$. The expression for $\Lambda$, Eq. (36), suggests that the splitting can be tuned by the external magnetic field. The linewidth of those two resonances is defined by $\alpha^{\prime}$ and $\beta$, and the shape is a combination of the symmetric and antisymmetric Lorentzians as in Fig. 4. Let us make estimates for a system shown in Fig. 6. The dimensions of the metallic links are chosen here as $(0.5 \times 0.05 \times 0.01) \mu \mathrm{m}$ with a Py load of the size $(0.1 \times 0.2$ $\times 0.02) \mu \mathrm{m}$, for which we can estimate a resonance frequency in the range of $0.5 \mathrm{GHz}(\mu \sim 100 \mathrm{GPa})$ (Ref. 20) and a coupling parameter $g \sim 0.001$ (free-standing metallic structures of such dimensions have already been realized ${ }^{21}$ ). For intermetallic interfaces, the "effective field effect" due to $\eta_{2}$ is very small, thus the phase shift of the magnetization is absent $\left(\Gamma_{y}=0\right)$. The amplitudes, given by Eqs. (22) and (23), are large in the proximity of the ferromagnetic resonance (FMR). The magnetovibrational coupling splits the FMR peak by $\sqrt{g} \omega\left(H_{0}<N_{x} M_{s}\right)$, which allows an electric detection of the mechanical motion excited by the spin transfer. Nor-

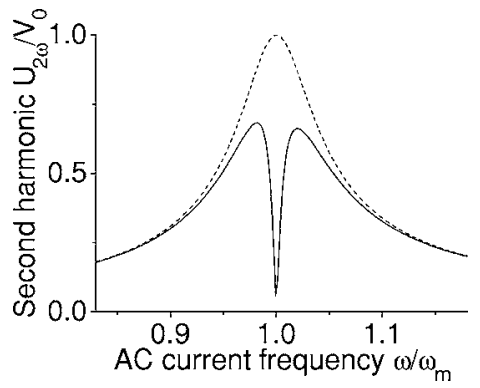

FIG. 8. Dependence of the second harmonic of the voltage on the frequency of the ac current bias for purely FMR (dashed line) and MVR (solid line) $\left(\omega_{m}=\omega_{e}, \alpha^{\prime}=0.02, \beta / \omega=0.002, g=0.001\right)$.

malized voltages are plotted in Figs. 7 and 8 for purely ferromagnetic (mechanical motion is suppressed) compared to magnetovibrational (MVR) resonances.

Finally, we write the response function for arbitrary magnetization directions in Fig. 3,

$$
\begin{gathered}
\chi_{y^{\prime} I}(\omega)=\left(M_{y^{\prime}} / I\right)_{\omega}=\frac{\hbar \gamma \sin \theta}{2 e V_{m}} \\
\stackrel{g \rightarrow 0}{\approx} \frac{\hbar \sin \theta \gamma}{2 e V_{m}} \frac{-i \eta_{1} \omega+\gamma M_{s} \Gamma_{y^{\prime}}}{\omega^{2}-\omega_{m}+2 i \alpha^{\prime} \omega \omega_{m}+\Lambda F(\omega)},
\end{gathered}
$$

where $\Lambda$ is defined in Eq. (40). As one can see, the form of the response function does not change. Equation (38) can be substituted into the first parts of Eqs. (22) and (23) in order to calculate the nonlinear response to an ac current. Similarly to Eq. (16), the parameter $\Gamma_{y^{\prime}} \approx \eta_{1} N_{x^{\prime} y^{\prime}}+\eta_{2} N_{x^{\prime}}$ governs the balance between the symmetric and antisymmetric Lorentzians composing each resonance (see Fig. 4). The magnetovibrational coupling is described by $\Lambda$,

$$
\begin{gathered}
\operatorname{Re} \Lambda=g\left[\omega^{2}-\left(H_{z^{\prime}}+\cot \theta_{1} H_{y^{\prime \prime}}\right) \omega_{m} /\left(N_{x^{\prime \prime}} M_{s}\right)\right], \\
\operatorname{Im} \Lambda=g \omega \gamma\left[H_{z^{\prime}} N_{x^{\prime \prime} y^{\prime \prime}} / N_{x^{\prime \prime}}+\cot \theta_{1}\left(H_{x^{\prime \prime}}+H_{y^{\prime \prime}} N_{x^{\prime \prime} y^{\prime \prime}} / N_{x^{\prime \prime}}\right)\right],
\end{gathered}
$$

and

$$
\begin{aligned}
g & =\sin ^{2} \theta_{1} M_{s}^{2} V_{m} N_{x^{\prime \prime}}[1 /(C k \cot (k L))] \\
& \approx \sin ^{2} \theta_{1} M_{s}^{2} V_{m} N_{x^{\prime \prime}} L / C=\sin ^{2} \theta_{1} N_{x^{\prime \prime}}(L / a)^{2}\left(V_{m} / V_{l}\right)\left(M_{s}^{2} / \mu\right),
\end{aligned}
$$

where $V_{m}$ and $V_{l}$ are the volumes of the magnetic load and the normal metal spring, respectively. $L$ and $a$ are the largest and the smallest dimensions of the normal metal links, $k$ $=\omega_{e} / c, \theta_{1}$ is the angle between the equilibrium direction of the magnetization $\mathbf{M}_{2}$ and the current flow, and the external field is $\mathbf{H}_{0}=\left(H_{x^{\prime \prime}}, H_{y^{\prime \prime}}, H_{z^{\prime}}\right)$. The parameter $\Lambda$ is calculated in a reference frame $x^{\prime \prime}, y^{\prime \prime}, z^{\prime}$ shown in Fig. 3. The $x^{\prime \prime}$ axis is perpendicular to the current flow and the axis $z^{\prime}$ (in general, this reference frame is different from the one used in Sec. III A, since the $x^{\prime \prime}$ axis is not necessarily along the direction of $\mathbf{m}_{1} \times \mathbf{m}_{2}$ ). 


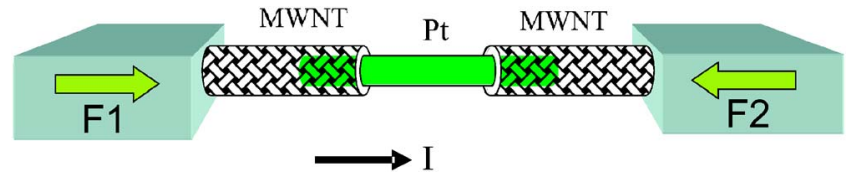

FIG. 9. (Color online) A spin-transfer nanomotor in which a metallic wire (rotor) with strong spin-flip scattering (such as $\mathrm{Pt}$ ) is grown inside a MWNT that is connected to two ferromagnetic contacts.

\section{Mechanical torques due to absorption of transversely polarized currents}

Let us now apply a dc current bias to the system in Fig. 6 . According to the magnetoelastic equations discussed above, the angular momentum of the spin-polarized current is completely transformed into mechanical torques when the crystal and shape anisotropies are strong enough to prevent magnetization motion relative to the lattice. The mechanical torque then equals the spin-transfer torque,

$$
T_{s t}=-\gamma \frac{\hbar}{2 e} I \eta_{1} \mathbf{m} \times\left(\mathbf{m}_{\text {fixed }} \times \mathbf{m}\right) \approx-\gamma T_{0} \eta_{1} .
$$

The Gilbert damping does not appear explicitly in this formula; however, it determines the time scale $1 /\left(\alpha \gamma M_{s}\right)$ in which the system reaches quasiequilibrium. The upper boundary for the spin-transfer-induced mechanical torque for a thin film of the size $(20 \times 200 \times 200) \mathrm{nm}$ (without crystal anisotropy) is defined by the maximum effective magnetic field due to the form anisotropy, $H_{\text {eff }}=4 \pi \theta M_{s}$, where $\theta$ $\approx \pi / 20 \sim H_{0} / M_{s}$ corresponds to some small stationary deflection of the magnetization out of the plane of the film in the presence of the stabilizing external magnetic field $\mathbf{H}_{0}$ (Fig. 6). The effective field generates a torque of $\gamma 4 \pi \theta M_{s} M_{s} V_{m} / \gamma \sim 10^{-16} \mathrm{~N} \mathrm{~m}$, where $V_{m}$ is the volume of the ferromagnet and $M_{s}=10^{6} \mathrm{~A} / \mathrm{m}$ is the saturation magnetization for Py. Such torques are well above the sensitivity of existing NEMS oscillators. ${ }^{22}$

\section{SPIN-TRANSFER NANOMOTOR}

Finally, we address the question how the torque can be transformed into a potentially useful rotary motion. It has been suggested in the literature to use carbon nanotubes as bearings for metallic nanowires. ${ }^{1,3}$ In Fig. 9, we propose a design of a spin-transfer nanomotor based on a multiwall carbon nanotube (MWNT) connected to two ferromagnetic electrodes (the torque doubles when the second electrode is ferromagnetic, but one ferromagnet is sufficient in principle). A metallic nanowire with a strong spin flip scattering is encapsulated by the MWNT. Pt would be a good choice $\left[l_{s d}^{\mathrm{Pt}}\right.$ $\sim 20 \mathrm{~nm}$ at $4.2 \mathrm{~K}$ (Ref. 23) and $l_{s d}^{\mathrm{Pt}} \sim 1 \mathrm{~nm}$ at room temperature ${ }^{24}$. FeCo nanowires that have already been grown inside nanotubes, ${ }^{25}$ presumably have spin-flip diffusion lengths not much different from FeNi $\left(l_{s d}^{\mathrm{Py}} \sim 5 \mathrm{~nm}\right),{ }^{26}$ which makes this material also very suitable. The metallic nanowire should preferably be longer than the spin-diffusion length in order to achieve a complete angular momentum transfer. After burning off the outer shells over the platinum nanowire (in addition, the MWNT may also be pulled out to open the Pt wire), we force the current to flow through the metallic wire that serves as a spin-sink and a rotor. It has recently been calculated that the conductance may rapidly oscillate due to quantum interference effects as we change the overlap between two nanotube shells, ${ }^{27-29}$ but disorder strongly enhances the intershell conductance, consistent with experiments. ${ }^{30}$

The situation with not too high polarization of the MWNT connection to a ferromagnet ${ }^{31}(P \sim 0.01)$ has a tendency to improve with recent experiments reporting $\mathrm{TMR}=5 \%(P$ $\sim 0.2) .{ }^{38-40}$ Half-metallic contacts to nanotubes might lead to much higher values. ${ }^{41}$ Adopting the maximum current through a single MWNT measured to date, ${ }^{34} I=1 \mathrm{~mA}$, we arrive at an estimate for the mechanical torque generated in the rotor $T \sim T_{0} \simeq 10^{-19} \mathrm{~N} \mathrm{~m}$ where we optimistically assume $P \sim 1$. This exceeds by many orders of magnitude the torques that can be induced by circularly polarized light ( $T$ $\left.\sim 10^{-29} \mathrm{~N} \mathrm{~m}\right){ }^{3}$ The advantage of a MWNT bearing is that the friction force can be very small. The bearing may still get stuck at certain preferred positions that minimize the interaction energy of the sleeve and shaft. However, each layer is likely to have a different chirality, so the potential barrier (static friction force) hindering rotation should be very small. An upper boundary for the static friction force per unit area has been measured ${ }^{32}$ to be $6.6 \times 10^{-13} \mathrm{~N} / \mathrm{nm}^{2}$ (but the actual value is possibly much smaller). For a nanotube radius of $2 \mathrm{~nm}$, this corresponds to a static friction torque per unit area of less than $10^{-21} \mathrm{~N} \mathrm{~m} / \mathrm{nm}^{2}$. For the same nanotube radius, the overlap length between the rotor and the outer shells can be made at least $10 \mathrm{~nm}$ without the risk that the rotor gets stuck by the static friction. When the barrier to rotation is overcome, the static friction is taken over by the dynamic friction. The latter is much smaller initially, but increases proportional to the angular velocity. For temperature as low as $100 \mathrm{~K}$ the thermal energy is estimated to be much larger than the corrugation energy against rotation. ${ }^{33}$ Our nanomotor should spin with a rotational frequency in the range of $\mathrm{GHz}$ at room temperature, based on estimates for the dynamical friction of $10^{-30} \mathrm{NM} / \mathrm{nm}^{2} / \mathrm{Hz}^{33}$

The proposed motor could be useful in the next generation of synthetic nanometer-scale electromechanical systems. With an attached metal plate, the rotor can serve as a mirror, with relevance to high-density switching devices. ${ }^{1}$ The motor can find applications for inducing and detecting motion in microfluidics systems and biological systems.

\section{CONCLUSIONS AND OUTLOOK}

We find that the electric current-induced creation and detection of mechanical torques in magnetic nanostructures is possible by the rectification technique suggested recently in Refs. 9 and 10. We develop a detailed theory of the magnetization dynamics in the presence of electric currents relevant for these experiments that agrees with the very recent study by Kupferschmidt et al. ${ }^{35}$ Subsequently, we predict that an alternating current can drive magnetovibrational dynamics, which can be read out by the generated dc voltage. Finally, we come to the conclusion that electric current-induced me- 
chanical torques can create rotary motion. We propose a spin-transfer driven nanomotor based on integrating metallic nanowires with carbon nanotubes.

In the study of the rectification effect, we find that the dc voltage originates from two mechanisms: the rectification of the applied ac current and the spin-pumping by the precessing ferromagnet. The second mechanism is important only in very thin ferromagnetic layers when the Gilbert damping is strongly enhanced (in accordance with Ref. 35). It should be noted that the shape of dc voltage as a function of frequency is a symmetric Lorentzian for the spin-pumping mechanism. This can be distinguished from the voltage induced in the presence of noncollinear magnetic anisotropies or the "effective" spin-transfer field, which causes asymmetric line shapes.

We generalized these results to treat magnetovibrationally coupled systems. The strongest coupling is achieved when the lowest mechanical mode is at resonance with the FMR frequency. In that case, a magnetopolariton is formed, and the Lorentzian shape of the dc voltage splits by an amount that is governed by the sensitivity of the mechanical system to external torques and by the magnetic anisotropies (without which there would be no magnetovibrational coupling). The technique based on the resonant magnetovibrational coupling can also be used to detect vibrations that are created externally. It can therefore be an alternative to the magnetomotive technique employed in fast transducers of mechanical motion. $^{22}$

We conclude that the functionalities of the spin-transfer torque, already used in applications such as magnetic memories, can be extended by taking into account the coupling with the mechanical degrees of freedom. The experimental realization is a challenge, since free-standing metallic small structures on a micro and nanoscale need to be fabricated and manipulated.

\section{ACKNOWLEDGMENTS}

We thank Yaroslav Tserkovnyak, Xuhui Wang, and Yuli Nazarov for stimulating discussions. We appreciate helpful communications with Dan Ralph and Jack Sankey. We are grateful that Piet Brouwer sent us a manuscript (Ref. 35), reminding us of the importance of spin pumping. This work has been supported by the Dutch FOM Foundation, National Science Foundation Grant No. PHY99-07949, the EU Commission FP6 NMP-3 project 505587-1"SFINX," and the Research Council of Norway Grant No. 162742/V00.

\section{APPENDIX A: EFFECT OF MIXING CONDUCTANCE ON THE TORQUE AND MAGNETORESISTANCE}

In this appendix, we generalize the relations derived in Ref. 14 for the torques and resistances in a general $\mathrm{N}|\mathrm{F} 1| \mathrm{N}|\mathrm{F} 2| \mathrm{N}$ multilayered structures without bulk layer spinflip scattering to the presence of an imaginary part of the mixing conductances or "effective field." We use magnetoelectronic circuit theory as formulated by Eqs. (11) and (12). Our result for the spin-transfer (ST) torque acting on the first ferromagnet (the torque on the second ferromagnet can be obtained by permutation of indexes 1 and 2) can be summarized by

$$
\mathbf{T}_{\mathrm{ST}}^{1}=\frac{I \hbar}{2 e}\left[\eta_{s} \mathbf{m}_{1} \times\left(\mathbf{m}_{2} \times \mathbf{m}_{1}\right)+\eta_{f}\left(\mathbf{m}_{2} \times \mathbf{m}_{1}\right)\right]
$$

where we introduced the spin-transfer efficiencies

$$
\begin{aligned}
& \eta_{s}=\frac{\left[\left[R_{2-}\left(R_{r}+R_{1}\right)-R_{1-} R_{2} \alpha\right]\left(1+\frac{R_{2 r}}{R_{r}} \widetilde{G}_{1 i}^{2}\right)+\frac{R_{1 r}}{R_{r}}\left[\frac{R_{2-}}{G_{1}}-\frac{R_{1-}}{G_{2}} \alpha\right] \widetilde{G}_{2 i}^{2}+\frac{R_{1 r} R_{2 r}}{R_{r}}\left(R_{1-}+R_{2-} \alpha\right) \widetilde{G}_{1 i} \widetilde{G}_{2 i}\right]}{\left(\left(R_{r}+R_{1}\right)\left(R_{r}+R_{2}\right)-R_{1} R_{2} \alpha^{2}+\left[\frac{\left(R_{r}+R_{1}\right)}{G_{2}}-\frac{R_{2}}{G_{1}} \alpha^{2}\right] \frac{R_{2 r}}{R_{r}} \widetilde{G}_{1 i}^{2}+\left[\frac{\left(R_{r}+R_{2}\right)}{G_{1}}-\frac{R_{1}}{G_{2}} \alpha^{2}\right] \frac{R_{1 r}}{R_{r}} \widetilde{G}_{2 i}^{2}+2 \alpha \frac{R_{1 r} R_{2 r}}{R_{r}}\left(\frac{1}{G_{1}}+\frac{1}{G_{2}}\right) \widetilde{G}_{1 i} \widetilde{G}_{2 i}\right)}, \\
& \eta_{f}=\frac{\left(\left[R_{2-}\left(R_{r}+R_{1}\right)-R_{1-} R_{2} \alpha\right] \frac{R_{1 r}}{R_{r}} \widetilde{G}_{1 i}-\left[R_{1-}\left(R_{r}+R_{2}\right)-R_{2-} R_{1} \alpha\right] \frac{R_{2 r}}{R_{r}} \widetilde{G}_{2 i}+\frac{R_{1 r}}{R_{r}}\left[\frac{R_{2-}}{G_{1}}-\frac{R_{1-}}{G_{2}} \alpha\right] \widetilde{G}_{1 i} \widetilde{G}_{2 i}^{2}-\frac{R_{2 r}}{R_{r}}\left[\frac{R_{1-}}{G_{2}}-\frac{R_{2-}}{G_{1}} \alpha\right] \widetilde{G}_{2 i} \widetilde{G}_{1 i}^{2}\right)}{\left(\left(R_{r}+R_{1}\right)\left(R_{r}+R_{2}\right)-R_{1} R_{2} \alpha^{2}+\left[\frac{\left(R_{r}+R_{1}\right)}{G_{2}}-\frac{R_{2}}{G_{1}} \alpha^{2}\right] \frac{R_{2 r}}{R_{r}} \widetilde{G}_{1 i}^{2}+\left[\frac{\left(R_{r}+R_{2}\right)}{G_{1}}-\frac{R_{1}}{G_{2}} \alpha^{2}\right] \frac{R_{1 r}}{R_{r}} \widetilde{G}_{2 i}^{2}+2 \alpha \frac{R_{1 r} R_{2 r}}{R_{r}}\left(\frac{1}{G_{1}}+\frac{1}{G_{2}}\right) \widetilde{G}_{1 i} \widetilde{G}_{2 i}\right)},
\end{aligned}
$$

with $\quad \alpha=\cos \theta, \quad 4 R_{1(2)}=1 / G_{1(2) \uparrow}+1 / G_{1(2) \downarrow}-2 / G_{1(2) r}, \quad 4 R_{1(2)-}=1 / G_{1(2) \uparrow}-1 / G_{1(2) \downarrow}, \quad 4 / G_{1(2)}=1 / G_{1(2) \uparrow}+1 / G_{1(2) \downarrow}, \quad 2 R_{1(2) r}$ $=1 / G_{1(2) r}, 2 R_{r}=1 / G_{1 r}+1 / G_{2 r}$, and $\widetilde{G}_{1(2) i}=G_{1(2) i} / G_{1(2) r}$, where $G_{1(2) \uparrow}$ and $G_{1(2) \downarrow}$ are conductances of the left (right) ferromagnet including the left (right) normal layer (when necessary, the middle layer conductance can also be included in these conductances), and $G_{1(2) \uparrow \downarrow}=G_{1(2) r}+i G_{1(2) i}$ is the mixing conductance of the left (right) ferromagnet.

The angular magnetoresistance reads

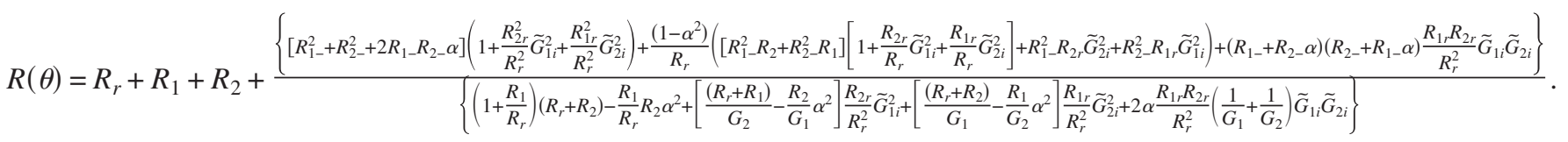




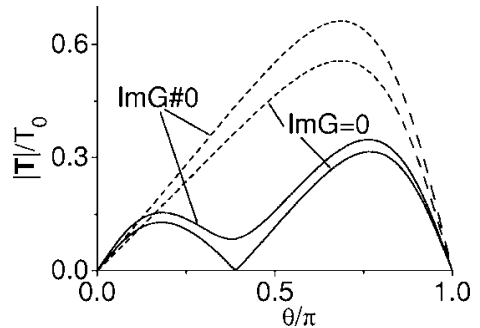

FIG. 10. Enhancement of the torque by a nonzero imaginary part of the spin-mixing conductance; dashed line for symmetric ( $\left.P=R_{-} G=0.7, G / G_{r}=0.6, \widetilde{G}_{i}=0.6\right)$ and solid line for asymmetric junctions $\left(P_{1}=R_{1-} G_{1}=0.7, G_{1} / G_{1 r}=G_{1} / G_{2 r}=0.6, \widetilde{G}_{1 i}=\widetilde{G}_{2 i}=0.4\right.$, $G_{2}=2 G_{1}, P_{2}=0.2$ ).

When the imaginary part of the mixing conductance is small, the absolute value of the torque Eq. (A1) and the magnetoresistance Eq. (A2) contain only second-order corrections in $\widetilde{G}_{1 i}$ and $\widetilde{G}_{2 i}$. In general, we conclude that the torque is enhanced when the imaginary part is not zero, as is shown in Fig. 10. This enhancement makes it impossible to have noncollinear points of zero torque reported earlier ${ }^{14,42}$ (see Fig. 10), which can influence the stable precessional states.

\section{APPENDIX B: SPIN PUMPING AND dc VOLTAGE IN ASYMMETRIC N|F|N STRUCTURES}

We consider here an asymmetric N1|F|N2 layered structure connected to two reservoirs and excited by rf magnetic fields. We are interested in the dc voltage that builds up under the condition of zero charge current. The system can be described by the generalized LLG equation and magnetoelectronic circuit theory,

$$
\begin{gathered}
\frac{d \mathbf{M}}{d t}=-\gamma \mathbf{M} \times \mathbf{H}_{\mathrm{eff}}+\frac{\alpha}{M_{s}} \mathbf{M} \times\left(\frac{d \mathbf{M}}{d t}\right) \\
-\frac{\gamma \hbar}{2 e V_{m}} \mathbf{m} \times\left[\left(\mathbf{I}_{s 1}+\mathbf{I}_{s 2}\right) \times \mathbf{m}\right], \\
I_{1(2)}=\left(G_{\uparrow}+G_{\downarrow}\right)\left(\mu_{0}^{2(1)}-\mu_{0}^{1(2)}\right)+\left(G_{\uparrow}-G_{\downarrow}\right)\left(\boldsymbol{\mu}_{s}^{2(1)}-\boldsymbol{\mu}_{s}^{1(2)}\right) \cdot \mathbf{m}, \\
\mathbf{I}_{s 1(2)}=\mathbf{m}\left[\left(G_{\uparrow}-G_{\downarrow}\right)\left(\mu_{0}^{2(1)}-\mu_{0}^{1(2)}\right)+\left(G_{\uparrow}+G_{\downarrow}\right)\left(\boldsymbol{\mu}_{s}^{2(1)}\right.\right. \\
\left.\left.-\boldsymbol{\mu}_{s}^{1(2)}\right) \cdot \mathbf{m}\right]-\left(2 \mathbf{m} \times \boldsymbol{\mu}_{s}^{1(2)}-\hbar \dot{\mathbf{m}}\right) \times \mathbf{m} G_{r}-(2 \mathbf{m} \\
\left.\times \boldsymbol{\mu}_{s}^{1(2)}-\hbar \dot{\mathbf{m}}\right) G_{i},
\end{gathered}
$$

where $G_{\uparrow}$ and $G_{\downarrow}$ describe the spin-dependent conductance limited by interface and bulk scattering of the ferromagnet, $G_{\uparrow \downarrow}=G_{r}+i G_{i}$ is the interface mixing conductance of the ferromagnet, the vector $\mathbf{m}=\mathbf{M} / M_{s}$ is the direction of the magnetization, and $\mu_{0}$ and $\boldsymbol{\mu}_{s}$ are the chemical potential and spin-accumulation in the normal metals, respectively.

For metallic interfaces, $G_{i}$ usually amounts to only a few percent of $G_{r}$. We calculate here the linear response to the external $\mathrm{rf}$ magnetic field when $G_{i}$ is disregarded,

$$
\begin{aligned}
& \chi_{x x}(\omega)=\left(M_{x} / h_{x}\right)_{\omega}=\frac{\left(\gamma M_{s}\right)^{2}\left(N_{y}+N_{x y} \alpha\right)}{\omega^{2}\left(1+\alpha_{s e}^{2}\right)-\omega_{m}^{2}\left(1+\alpha^{2}\right)+2 i \alpha^{\prime} \omega \omega_{m}}, \\
& \chi_{y x}(\omega)=\left(M_{y} / h_{x}\right)_{\omega}=\frac{\gamma M_{s}\left[\gamma M_{s}\left(N_{x y}+N_{x} \alpha\right)-i \omega\right]}{\omega^{2}\left(1+\alpha_{s e}^{2}\right)-\omega_{m}^{2}\left(1+\alpha^{2}\right)+2 i \alpha^{\prime} \omega \omega_{m}},
\end{aligned}
$$

where $\alpha$ is the bulk Gilbert damping, $\alpha_{s e}=\gamma \hbar^{2}\left(G_{1}\right.$ $\left.+G_{2}\right) /\left(2 e M_{s} V_{m}\right)$ is the extra damping due to spin emission, $1 / G_{1}=1 / g_{1}+1 /\left(2 G_{r}\right)$, and $1 / G_{2}=1 / g_{2}+1 /\left(2 G_{r}\right)$ with $g_{1}$ and $g_{2}$ being conductances of the R1|N1 and N2|R2 interfaces, respectively, $\quad \alpha^{\prime}=\left(\alpha+\alpha_{s e}\right)\left(N_{x}+N_{y}\right) /\left(2 \sqrt{N_{x} N_{y}-N_{x y}^{2}}\right) \quad$ (note that, when important, bulk scattering can be easily included into the conductances $g_{1}, g_{2}$, and $\left.G_{r}\right) .{ }^{14,43}$

By solving Eqs. (B1)-(B3) to the second order in a small rf magnetic field, the generated dc voltage can be expressed via the susceptibilities Eqs. (B4) and (B5) as

$$
e V=2 \hbar \omega P G_{r}\left(\frac{1}{g_{1}}-\frac{1}{g_{2}}\right) \operatorname{Im}\left(\chi_{x x} \chi_{y x}^{*}\right) h_{x}^{2} / M_{s}^{2},
$$

where we introduced the effective polarization of the device $P=\left(1 / G_{\uparrow}-1 / G_{\downarrow}\right) /\left(1 / G_{\uparrow}+1 / G_{\downarrow}+4 / g_{1}+4 / g_{2}\right)$. The asymmetry of the interfaces R1|N1 and N2|R2 is seen to be crucial for the generation of a dc voltage in this geometry. Note that the dependence of the dc voltage on the frequency has a Lorentzian line shape as follows from Eqs. (B4)-(B6). When $P>0$, the positive sign of the voltage corresponds to the voltage applied to the junction with higher conductance $g_{1(2)}$.

The first-order correction in the mixing conductance $G_{i}$ leads to a slight change of the height of the Lorentzian and to a shift of the resonance frequency,

$$
\omega^{2}=\omega_{m}^{2}\left(1-\frac{\gamma \hbar^{2}\left(G_{1}^{2}+G_{2}^{2}\right)}{4 e M_{s} V_{m}} \frac{G_{i}}{G_{r}^{2}}\right) .
$$

Note that the magnetovibrational coupling does not change the form Eq. (B6) when the proper susceptibilities Eqs. (34) are substituted. The magnetovibrational coupling can thus be observed as a splitting of the Lorentzian peak due to spin pumping in an asymmetric N1|F|N2 structure. Generation of a dc voltage by magnetization precession of a single ferromagnetic layer has been suggested in Ref. 37. However, those authors concentrate on the generation of a dc voltage due to spin-flip scattering in the ferromagnet.

\section{APPENDIX C: SPIN PUMPING AND RECTIFICATION OF ac CURRENTS IN F|N|F|N STRUCTURES}

It follows from the precedings appendix on $\mathrm{N} 1|\mathrm{~F}| \mathrm{N} 2$ structures that a spin-coherent $\mathrm{F} 1|\mathrm{~N}| \mathrm{F} 2 \mid \mathrm{N}$ structure, in which the normal metals are identical and an extra ferromagnetic layer F1 (with fixed magnetization) causes the asymmetry, should generate a dc voltage as well. In such a structure, ac currents instead of the rf magnetic field can generate spintransfer torques when the magnetizations are noncollinear. 
Since the analytical expressions for arbitrary angles between the magnetizations are complex, we concentrate here, without loss of generality, on a 90 degree configuration.

We assume a constant ac current bias on the system, thus forcing the extra charge current due to spin pumping to vanish. Following the derivation in Appendix B, and disregarding the imaginary parts of mixing conductances for both interfaces, we arrive at the following expressions for the linearresponse functions:

$$
\begin{aligned}
& \chi_{x I}(\omega)=\frac{\hbar \gamma}{2 e V_{m}} \frac{\eta_{1}\left[\gamma M_{s}\left(N_{y}+N_{x y} \alpha\right)-i \omega \alpha_{x}^{s e}\right]}{\omega^{2}\left(1+\alpha_{x}^{s e} \alpha_{y}^{s e}\right)-\omega_{m}^{2}\left(1+\alpha^{2}\right)+i \omega \Delta}, \\
& \chi_{y I}(\omega)=\frac{\hbar \gamma}{2 e V_{m}} \frac{\eta_{1}\left[\gamma M_{s}\left(N_{x y}+N_{x} \alpha\right)-i \omega\right]}{\omega^{2}\left(1+\alpha_{x}^{s e} \alpha_{y}^{s e}\right)-\omega_{m}^{2}\left(1+\alpha^{2}\right)+i \omega \Delta},
\end{aligned}
$$

where the anisotropic Gilbert dampings due to spin emission are $\quad \alpha_{x}^{s e}=\gamma \hbar^{2}\left(G_{1}+G_{2}\right) /\left(2 e M_{s} V_{m}\right), \quad \alpha_{y}^{s e}=\gamma \hbar^{2}\left(G_{\uparrow \downarrow}\right.$ $\left.+G_{2}\right) /\left(2 e M_{s} V_{m}\right), \quad \Delta=\gamma M_{s}\left[N_{x}\left(\alpha+\alpha_{x}^{s e}\right)+N_{y}\left(\alpha+\alpha_{y}^{s e}\right)+\alpha\left(\alpha_{y}^{s e}\right.\right.$ $\left.\left.-\alpha_{x}^{s e}\right) N_{x y}\right], \quad 1 / G_{1}=\left(1 / g_{1 \uparrow}+1 / g_{1 \downarrow}\right) / 4+1 /\left(2 G_{r}\right), \quad 1 / G_{2}=1 / g_{2}$ $+1 /\left(2 G_{r}\right)$, and $1 / G_{\uparrow \downarrow}=1 /\left(2 G_{r}\right)+1 /\left(2 g_{1 r}\right)$, with $g_{1 \uparrow \downarrow}=g_{1 r}$ $+i g_{1 i}$ (however $g_{1 i}$ is neglected) and $g_{1 \uparrow(\downarrow)}$ being the mixing and normal conductances of the R1|F interface, respectively.

The second-order analysis provides us with an expression for the dc voltage. After combining it with Eq. (22), we arrive at the full expression for the dc voltage in terms of the susceptibilities Eqs. (C1) and (C2), consisting of separate contributions due to rectification and spin pumping,

$$
\begin{aligned}
U_{0}= & \frac{I_{0}^{2}}{2 M_{s}} \frac{\partial R(\nu)}{\partial \nu} \operatorname{Re} \chi_{y I} \\
& +\frac{2 \hbar \omega}{e} \mathrm{PG}_{r}\left(\frac{1}{\widetilde{g}_{1}}-\frac{1}{g_{2}}\right) \operatorname{Im}\left(\chi_{x I} \chi_{y I}\right) I_{0}^{2} / M_{s}^{2},
\end{aligned}
$$

where an effective conductance is $1 / \tilde{g}_{1}=\left[3 /\left(2 g_{1 r}\right)\right.$ $\left.+\left(1 /\left(4 g_{1 \uparrow}\right)+1 /\left(4 g_{1 \downarrow}\right)\right) R_{\uparrow \downarrow} / R_{1}\right] / 4$ and an effective polarization of the device is $P=\left(1 /\left(4 G_{\uparrow}\right)-1 /\left(4 G_{\downarrow}\right)\right) /\left(1 /\left(4 G_{\uparrow}\right)\right.$ $\left.+1 /\left(4 G_{\downarrow}\right)+1 /\left(2 g_{1 r}\right)+1 /\left(g_{2}\right)\right)$. The dependence of the first term in Eq. (C3) on the frequency is in general a combination of the Lorentzian with an antisymmetric Lorentzian, as is discussed in Sec. III A. The second term becomes important when $\alpha^{s e} \geq \alpha$ and $\left(1 / \tilde{g}_{1}-1 / g_{2}\right) /\left(1 / g_{1 \uparrow}-1 / g_{1 \downarrow}\right) \geq 1$. When the damping due to spin emission is small or the asymmetry of the trilayer weak, the second term in Eq. (C3) can be disregarded. Its dependence on the frequency has a Lorentzian shape; see Eqs. (C1) and (C2). In general, the signs of the first and second term in Eq. (C3) can be opposite, thus possibly suppressing the symmetric-Lorentzian part of dc voltage.

The first-order corrections in small mixing conductances, $G_{i}$ and $g_{1 i}$, lead to a change of the height of the symmetric and antisymmetric parts of Lorentzian and also to a shift of the resonance frequency, as it arises from the expressions for the susceptibilities,

$$
\chi_{x I}(\omega)=\frac{\hbar \gamma}{2 e V_{m}} \frac{i \omega\left(\eta_{2}-\eta_{1} \alpha_{x}^{s e}\right)+\gamma M_{s} \Gamma_{x}}{\omega^{2}\left(1+\alpha_{x}^{s e} \alpha_{y}^{s e}+2 \kappa\right)-\omega_{m}^{2}\left(1+\alpha^{2}\right)+i \omega \Delta},
$$

$$
\chi_{y I}(\omega)=\frac{\hbar \gamma}{2 e V_{m}} \frac{-i \omega\left(\eta_{1}+\eta_{1} \kappa-\eta_{2} \alpha_{y}^{s e}\right)+\gamma M_{s} \Gamma_{y}}{\omega^{2}\left(1+\alpha_{x}^{s e} \alpha_{y}^{s e}+2 \kappa\right)-\omega_{m}^{2}\left(1+\alpha^{2}\right)+i \omega \Delta},
$$

where $\kappa=\gamma\left(\hbar^{2} / 4 e M_{s} V_{m}\right)\left[\left(1 / R_{\uparrow \downarrow} R_{1}\right)+\left(1 / R_{2}^{2}\right)\right] \frac{G_{i}}{G_{r}^{2}}, \quad \Gamma_{x}=\eta_{1}\left(N_{y}\right.$ $\left.+N_{x y} \alpha\right)+\eta_{2}\left(N_{x y}-N_{y} \alpha\right)$, and $\Gamma_{y}=\eta_{1}\left(N_{x y}+N_{x} \alpha\right)+\eta_{2}\left(N_{x}\right.$ $\left.-N_{x y} \alpha\right)$.

Finally, we present our results in the presence of the magnetovibrational coupling (e.g., see Fig. 6) keeping only the dominant terms in small Gilbert damping,

$$
\begin{aligned}
\chi_{x I}(\omega)= & \frac{\hbar \sin \theta \gamma}{2 e V_{m}} \\
& \times \frac{i \omega\left[\eta_{2}-\eta_{1} \alpha_{x}^{s e}+g\left(1-N_{x y} / N_{x}\right) F(\omega)\right]+\gamma M_{s} \Gamma_{x}}{\omega^{2}(1+\kappa)-\omega_{m}^{2}+i \omega \Delta+\Lambda F(\omega)} \\
\stackrel{g \rightarrow 0}{\approx} & \frac{\hbar \sin \theta \gamma}{2 e V_{m}} \frac{\eta_{2} i \omega+\gamma M_{s} \Gamma_{x}}{\omega^{2}-\omega_{m}+i \omega \Delta+\Lambda F(\omega)},
\end{aligned}
$$

These susceptibilities can be used in Eq. (C3) in order to calculate the generated dc voltage. In the regime of resonant magnetovibrational coupling, the second (spin emission) term in Eq. (3) is comparable with the first one only when $\alpha^{s e} \sim \beta / \omega_{m}$. The second term corresponds to two symmetric
Lorentzian peaks split by $\operatorname{Re} \Lambda$.

Spin pumping therefore can play an important role when the enhanced interface damping is comparable to the bulk Gilbert damping and, in the regime of magnetovibrational coupling, the mechanical damping. 
${ }^{1}$ A. M. Fennimore, T. D. Yuzvinsky, W.-Q. Han, M. S. Fuhrer, J. Cumings, and A. Zettl, Nature (London) 424, 408 (2003).

${ }^{2} \mathrm{Z}$. Xu and Q. Zheng, cond-mat/0606302 (unpublished).

${ }^{3}$ P. Král and H. R. Sadeghpour, Phys. Rev. B 65, 161401(R) (2002).

${ }^{4}$ P. Mohanty, G. Zolfagharkhani, S. Kettemann, and P. Fulde, Phys. Rev. B 70, 195301 (2004).

${ }^{5}$ A. G. Mal'shukov, C. S. Tang, C. S. Chu, and K. A. Chao, Phys. Rev. Lett. 95, 107203 (2005).

${ }^{6}$ A. A. Kovalev, G. E. W. Bauer, and A. Brataas, Jpn. J. Appl. Phys., Part 1 45, 3878 (2006).

${ }^{7}$ M. D. Stiles and A. Zangwill, J. Appl. Phys. 91, 6812 (2002).

${ }^{8}$ A. A. Kovalev, G. E. W. Bauer, and A. Brataas, Appl. Phys. Lett. 83, 1584 (2003).

${ }^{9}$ A. A. Tulapurkar, Y. Suzuki, A. Fukushima, H. Kubota, H. Maehara, K. Tsunekawa, D. D. Djayaprawira, N. Watanabe, and S. Yuasa, Nature (London) 438, 339 (2005).

${ }^{10}$ J. C. Sankey, P. M. Braganca, A. G. F. Garcia, I. N. Krivorotov, R. A. Buhrman, and D. C. Ralph, Phys. Rev. Lett. 96, 227601 (2006).

${ }^{11}$ Y. Tserkovnyak, A. Brataas, and G. E. W. Bauer, Phys. Rev. Lett. 88, 117601 (2002).

${ }^{12}$ L. D. Landau and E. M. Lifshitz, Theory of Elasticity, 2nd ed. (Pergamon, Oxford, 1959).

${ }^{13}$ A. Brataas, Y. V. Nazarov, and G. E. W. Bauer, Eur. Phys. J. B 22, 99 (2001); A. Brataas, G. E. W. Bauer, and P. J. Kelly, Phys. Rep. 427, 157 (2006).

${ }^{14}$ A. A. Kovalev, A. Brataas, and G. E. W. Bauer, Phys. Rev. B 66, 224424 (2002).

${ }^{15}$ X. Wang, G. E. W. Bauer, and A. Hoffmann, Phys. Rev. B 73, 054436 (2006).

${ }^{16}$ X. Wang, G. E. W. Bauer, and T. Ono, Jpn. J. Appl. Phys., Part 1 45, 3863 (2006).

${ }^{17}$ J. C. Slonczewski, J. Magn. Magn. Mater. 159, L1 (1996).

${ }^{18}$ J. C. Slonczewski, Phys. Rev. B 71, 024411 (2005).

${ }^{19}$ X. Ming, H. Huang, C. A. Zorman, M. Mehregany and M. L. Roukes, Nature (London) 421, 496 (2003).

${ }^{20}$ M. G. Simmons and H. Wang, Single Crystal Elastic Constants and Calculated Aggregate Properties (MIT Press, Cambridge, MA, 1975).

${ }^{21}$ G. S. Paraoanu and A. M. Halvari, Appl. Phys. Lett. 86, 093101
(2005).

${ }^{22}$ X. M. H. Huang, X. L. Feng, C. A. Zorman, M. Mehregany, and M. L. Roukes, New J. Phys. 7, 247 (2005).

${ }^{23}$ S. K. Olson, R. Loloee, N. Theodoropoulou, W. P. Pratt, Jr., J. Bassa, P. X. Xu, and Ke Xia, Appl. Phys. Lett. 87, 252508 (2005).

${ }^{24}$ S. Mizukami, Y. Ando, and T. Miyazaki, Phys. Rev. B 66, 104413 (2002).

${ }^{25}$ A. L. Elías et al., Nano Lett. 5, 467 (2005).

${ }^{26}$ S. Urazhdin, R. Loloee, and W. P. Pratt, Jr., Phys. Rev. B 71, 100401(R) (2005).

${ }^{27}$ D.-H. Kim and K. J. Chang, Phys. Rev. B 66, 155402 (2002).

${ }^{28}$ R. Tamura, Y. Sawai, and J. Haruyama, Phys. Rev. B 72, 045413 (2005).

${ }^{29}$ M. A. Tunney and N. R. Cooper, cond-mat/0606410 (unpublished).

${ }^{30}$ J. Cumings and A. Zettl, Phys. Rev. Lett. 93, 086801 (2004).

${ }^{31}$ K. Tsukagoshi, B. W. Alphenaar, and H. Ago, Nature (London) 401, 572 (1999).

${ }^{32}$ J. Cumings and A. Zettl, Science 289, 602 (2000).

${ }^{33}$ J. Servantie and P. Gaspard, Phys. Rev. Lett. 97, 186106 (2006).

${ }^{34}$ S. Dohn, K. Mølhave, and P. Bøggil, Sens. Lett. 3, 300 (2005).

${ }^{35}$ J. N. Kupferschmidt, S. Adam, and P. W. Brouwer, Phys. Rev. B 74, 134416 (2006).

${ }^{36}$ G. E. W. Bauer, A. Brataas, Y. Tserkovnyak, and B. J. van Wees, Appl. Phys. Lett. 82, 3928 (2003).

${ }^{37}$ X. Wang, G. E. W. Bauer, B. J. van Wees, A. Brataas, and Y. Tserkovnyak, cond-mat/0608022 (unpublished).

${ }^{38}$ S. Sahoo, T. Kontos, J. Furer, C. Hoffmann, M. Gräber, A. Cottet, and C. Schönenberger, Nat. Phys. 1, 99 (2005).

${ }^{39}$ N. Tombros, S. J. van der Molen, and B. J. van Wees, Phys. Rev. B 73, 233403 (2006).

${ }^{40}$ H. T. Man, I. J. W. Wever, and A. F. Morpurgo, Phys. Rev. B 73, 241401(R) (2006).

${ }^{41}$ L. E. Hueso, G. Burnell, J. L. Prieto, L. Granja, C. Bell, D. J. Kang, M. Chhowalla, S. N. Cha, J. E. Jang, G. A. J. Amaratunga, and N. D. Mathur, Appl. Phys. Lett. 88, 083120 (2006).

${ }^{42}$ J. Manschot, A. Brataas, and G. E. W. Bauer, Phys. Rev. B 69, 092407 (2004).

${ }^{43}$ A. A. Kovalev, G. E. W. Bauer, and A. Brataas, Phys. Rev. B 73, 054407 (2006). 